\title{
Nanoscale
}

D) Check for updates

Cite this: Nanoscale, 2020, 12, 17450

\section{Relating the composition and interface interactions in the hard corona of gold nanoparticles to the induced response mechanisms in living cells $\uparrow$}

\author{
Gergo Peter Szekeres, (D) $\ddagger^{\mathrm{a}, \mathrm{b}}$ Stephan Werner, ${ }^{\mathrm{c}}$ Peter Guttmann, (D) \\ Cecilia Spedalieri, ${ }^{b}$ Daniela Drescher, ${ }^{b}$ Vesna Živanović, ${ }^{\mathrm{a}, \mathrm{b}}$ Maria Montes-Bayón, (D) a,d \\ Jörg Bettmer (D) ${ }^{d}$ and Janina Kneipp (D)*a,b
}

\begin{abstract}
Understanding the formation of the intracellular protein corona of nanoparticles is essential for a wide range of bio- and nanomedical applications. The innermost layer of the protein corona, the hard corona, directly interacts with the nanoparticle surface, and by shielding the surface, it has a deterministic effect on the intracellular processing of the nanoparticle. Here, we combine a direct qualitative analysis of the hard corona composition of gold nanoparticles with a detailed structural characterization of the molecules in their interaction with the nanoparticle surface and relate both to the effects they have on the ultrastructure of living cells and the processing of the gold nanoparticles. Cells from the cell lines HCT-116 and A549 were incubated with $30 \mathrm{~nm}$ citrate-stabilized gold nanoparticles and with their aggregates in different culture media. The combined results of mass spectrometry based proteomics, cryo soft $X$-ray nanotomography and surface-enhanced Raman scattering experiments together revealed different uptake mechanisms in the two cell lines and distinct levels of induced cellular stress when incubation conditions were varied. The data indicate that the different incubation conditions lead to changes in the nanoparticle processing via different protein-nanoparticle interfacial interactions. Specifically, they suggest that the protein-nanoparticle surface interactions depend mainly on the surface properties of the gold nanoparticles, that is, the $\zeta$-potential and the resulting changes in the hydrophilicity of the nanoparticle surface, and are largely independent of the cell line, the uptake mechanism and intracellular pro-

cessing, or the extent of the induced cellular stress.
\end{abstract}

Received 7th May 2020,

Accepted 25th July 2020

DOI: 10.1039/d0nr03581e

rsc.li/nanoscale

\section{Introduction}

With the emerging exposure of people, animals, and plants to nanomaterials it becomes essential to understand the intracellular processing of nanoparticles by their biomolecular

\footnotetext{
${ }^{a}$ Humboldt-Universität zu Berlin, School of Analytical Sciences Adlershof, AlbertEinstein-Str. 5-9, 12489 Berlin, Germany.

E-mail: janina.kneipp@chemie.hu-berlin.de

${ }^{b}$ Humboldt-Universität zu Berlin, Department of Chemistry, Brook-Taylor-Str. 2, 12489 Berlin, Germany

${ }^{c}$ Helmholtz-Zentrum Berlin für Materialien und Energie GmbH, Department X-ray Microscopy, Albert-Einstein-Str. 15, 12489 Berlin, Germany

${ }^{d}$ University of Oviedo, Department of Physical and Analytical Chemistry, Faculty of Chemistry and Instituto de Investigación Sanitaria del Principado de Asturias (ISPA), C/Julian Clavería 8, 33006 Oviedo, Spain

$\dagger$ Electronic supplementary information (ESI) available. See DOI: 10.1039/ d0nr03581e

\$Current address: Department of Molecular Physics, Fritz Haber Institute of the Max Planck Society, Faradayweg 4-6, 14195 Berlin, Germany.
}

environment. Nanoparticles have long been considered for biomedical use, since their surface is easily functionalized. When delivered into a complex biomolecular system, e.g., blood or living cells, their surface interacts with a multitude of protein molecules that form the protein corona. ${ }^{1}$ Cells can only see this protein corona, ${ }^{2}$ which triggers biomolecular response mechanisms different than pristine nanoparticles would, thus determining the processing of the nanomaterial in the biological system. ${ }^{3}$ Therefore, the protein corona plays a key role in the nanoparticle-induced cellular processes. The innermost layer of the protein corona, called the hard corona, consists of proteins with the strongest affinity to the nanoparticles' surface, while the outer protein layers interact with the hard corona proteins and with each other.

Most studies focus on the composition of the protein corona in complex protein solutions in vitro. ${ }^{1,4-7}$ The intracellular hard corona has so far been addressed mainly indirectly ${ }^{8}$ and as a corona complex including the outer protein layers as well. ${ }^{9}$ However, the knowledge of the hard 
protein corona formed in living cells, specifically of its composition, interfacial interactions, and its connection to nanoparticle processing in vivo is scarce but of crucial importance.

Here, the composition of the hard protein corona and its interaction with the nanoparticle surface in vivo are in focus. We combine proteomics by high-performance liquid chromatography-electrospray ionization tandem mass spectrometry (HPLC-ESI-Q-TOF-MS), ultrastructural information from cryo soft X-ray nanotomography (cryo-SXT) experiments, and the in vivo vibrational spectral information of the nanoparticleprotein interactions from surface-enhanced Raman scattering (SERS). Thereby it is possible to gain a comprehensive knowledge of the intracellular hard protein corona in the intact cells and to describe its influence on nanoparticle processing that is unprecedented by previous work where such approaches have been used separately. ${ }^{10-12}$

While HPLC-ESI-Q-TOF-MS reveals the composition of the hard corona, ${ }^{4,10}$ SERS can be used to determine protein-nanoparticle interactions. ${ }^{13,14}$ The SERS spectra are collected from living cells, as reported in other approaches. ${ }^{12,15,16}$ In this way, the interaction and composition of the hard protein corona as the immediate environment of the nanoparticles are probed in vivo. Cryo-SXT on vitrified cells of identical samples yields three-dimensional information with a resolution lower than $40 \mathrm{~nm}$, allowing for the detailed investigation of the localization and arrangement of the intracellular nanoparticle agglomerates, and the overall cellular ultrastructure in a quasinative state. ${ }^{11,17,18}$ The combination of these three types of information enables us to relate the processing of citratestabilized gold nanoparticles by the living cells to the composition of the intracellular hard protein corona and its dependence on properties of the nanoparticle surface in vivo. Here we address the influence of different culture media, two different epithelial cell lines, as well as applying gold nanoparticles in their pristine form or as aggregates, on the characteristics of the hard protein corona. We show that while the composition of the hard protein corona and the induced cellular response depend strongly on the cell line and the culturing conditions, respectively, the interaction of the hard protein corona with the nanoparticles depends only on the interfacial properties of the gold nanoparticles, regardless of variations in physiological aspects of their uptake.

The results pinpoint the influence of the inherent cellular processing and of the artificially designed physiological conditions on the composition of the hard protein corona and on the nanoparticle-protein interfacial interactions, which have further implications in biomedical research and in nanomedicine.

\section{Materials and methods}

\section{Gold nanoparticle synthesis}

$\sim 30 \mathrm{~nm}$ citrate stabilized gold nanoparticles $(30 \pm 7 \mathrm{~nm}$ calculated from 50 particles) were synthesized based on the protocol described by Lee and Meisel $^{19}$ and characterized by trans- mission electron microscopy and UV-vis spectroscopy as described previously, see Fig. S1. $\dagger^{20}$

\section{Cell cultivation and incubation}

Two epithelial cell lines, HCT-116 human colorectal carcinoma (LGC Standards, Wesel, Germany) and A549 human alveolar basal epithelial adenocarcinoma cells (DSMZ, Braunschweig, Germany), were grown under standard conditions $\left(37^{\circ} \mathrm{C}, 5 \%\right.$ $\mathrm{CO}_{2}$ ) in Dulbecco's modified Eagle medium supplemented with 10\% fetal bovine serum (both from Biochrom, Berlin, Germany) and in McCoy's modified 5A medium (SigmaAldrich, Steinheim, Germany) supplemented with $10 \%$ fetal bovine serum, respectively. When the cell culture reached 75-90\% confluence (depending on the experiment), the culture medium was exchanged with the $10: 1$ volume mixture of the respective culture medium and gold nanoparticles or gold nanoparticles mixed with $0.1 \mathrm{~mol} \mathrm{~L}^{-1} \mathrm{NaCl}$, yielding a final nominal gold nanoparticle concentration of $40 \mathrm{pmol} \mathrm{L}^{-1}$. After $24 \mathrm{~h}$, the culture medium with gold nanoparticles was removed, and the cells were rinsed with phosphate buffered saline (PBS, Biochrom, Berlin, Germany) to wash off excess gold nanoparticles. The incubation time of $24 \mathrm{~h}$ was determined based on prior experiments that indicated that the hard corona remains unchanged over the course of 24 h. ${ }^{10,21}$

\section{Analysis of the protein corona composition}

The protein corona composition was analyzed by HPLC-ESI-QTOF-MS as described previously. ${ }^{10}$ Briefly, 10000 000 cells were incubated in a $25 \mathrm{~cm}^{2}$ cell culture flask for the analysis of the protein corona composition. The cells underwent soft lysis upon addition of $150 \mu \mathrm{L}$ of lysis buffer containing $1 \%(\mathrm{v} / \mathrm{v})$ Triton X-100 as detergent, $150 \mathrm{mmol} \mathrm{L}^{-1} \mathrm{NaCl}, 1 \mathrm{mmol} \mathrm{L}^{-1}$ ethylenediaminetetraacetic acid, and $20 \mathrm{mmol} \mathrm{L}^{-1}$ tris(hydroxymethyl) aminomethane (all chemicals purchased from Sigma-Aldrich, Steinheim, Germany). After $5 \mathrm{~min}$ of incubation on ice, the nanoparticle-hard protein corona bioconjugates were purified through a sucrose cushion and three cycles of washing with PBS and centrifugation at $18000 \mathrm{~g}$, which was shown to eliminate the contribution of soft corona proteins. $^{4,10}$ After the separation of the corona proteins with gel electrophoresis and in-gel trypsinization, the protein fragments were identified with HPLC-ESI-Q-TOF-MS. The collected data were analyzed in MASCOT ${ }^{22}$ using the SwissProt database. The hard corona proteomes were analyzed by the Database for Annotation, Visualization and Integrated Discovery (DAVID) ${ }^{23,24}$ and STRING. ${ }^{25}$ DAVID was used to obtain protein groups with similar functions in the case of enriched proteins in the hard corona, while STRING analyzed them only based on function, and protein enrichment was not considered.

\section{Cryo soft X-ray nanotomography (cryo-SXT)}

Cells were grown on Formvar-coated gold tomography grids and cultured and incubated as described above. After rinsing three times with PBS to remove non-internalized gold nanoparticles, the excess liquid was blotted with a filter paper, and the sample was snap-frozen in liquid ethane with the help of a 
plunge freezer. Cryo-SXT experiments were performed with a transmission X-ray microscope equipped with a cryostage at the U41-XM beamline at the BESSY II electron storage ring (Helmholtz-Zentrum Berlin für Materialen und Energie, Berlin, Germany), which allowed for the native-state study of the cells. ${ }^{11,26}$ All images were taken at $510 \mathrm{eV}$ photon energy with a zone plate with an outermost zone width of $25 \mathrm{~nm}$. The angular range of the tilt series was between $-60^{\circ}$ and $+60^{\circ}$ with an angular step of $1^{\circ}$. Tomograms were reconstructed in Etomo (IMOD ${ }^{\odot}$ 4.9.0.) using the internalized gold nanoparticles as fiducial markers. Since the image pixel size corresponds to $\sim 9.8 \mathrm{~nm}$ per pixel, $30 \mathrm{~nm}$ gold nanoparticles are visible even as single particles in the cellular compartments. The 3D ultrastructure of the cells and the intracellular nanoparticle aggregates were segmented and visualized using Amira (Thermo-Fisher Scientific) software.

\section{Surface-enhanced Raman scattering (SERS)}

Cells were grown on glass coverslips and, after $24 \mathrm{~h}$ incubation with nanoparticles and subsequent rinsing with PBS, the slides were transferred to a Raman microspectrometer equipped with a nitrogen-cooled charge-coupled device detector (Horiba, Munich, Germany). A 60× water immersion objective (Olympus, Germany) was used for Raman mapping experiments of single cells. Each spectrum was acquired in $1 \mathrm{~s}$ with excitation by a $785 \mathrm{~nm}$ laser at a laser intensity of $2.3 \times 10^{5} \mathrm{~W} \mathrm{~cm}^{-2}$.

The spectra were frequency-calibrated using a spectrum of $50-50 \%$ toluene and acetonitrile. Spectral pre-processing in MatLab R2016b (The MathWorks, Inc.) comprised removal of spikes, background-correction using AsLS algorithm, ${ }^{27}$ and vector-normalization. Band occurrences were analyzed in Mathematica 11.0 (Wolfram). To analyze frequencies of occurrence of spectral bands, a band was considered as present, if the local integral exceeded the average integral value of all the $20 \mathrm{~cm}^{-1}$ windows of an identically pre-processed spectrum without signals and their standard deviation by at least a factor of five.

\section{Results and discussion}

The hard corona contains proteins important for nanoparticle processing

Two different cell lines with similar function, the epithelial cells A549 and HCT-116, were chosen for all experiments because epithelia are the first contact point of nanoparticles in an organism. After HCT-116 and A549 cells were respectively incubated with gold nanoparticles for $24 \mathrm{~h}$ in Dulbecco's modified Eagle's medium supplemented with $10 \%$ fetal bovine serum (DMEM-FBS), the hard protein corona of the internalized gold nanoparticles was purified and analyzed by gel electrophoresis and mass spectrometry. The tentative functional assignment of the hard corona proteins extracted from live cells provides an unprecedented insight into the interactions of the gold nanoparticles from the moment of their introduction into the cell culture medium until the cell lysis.

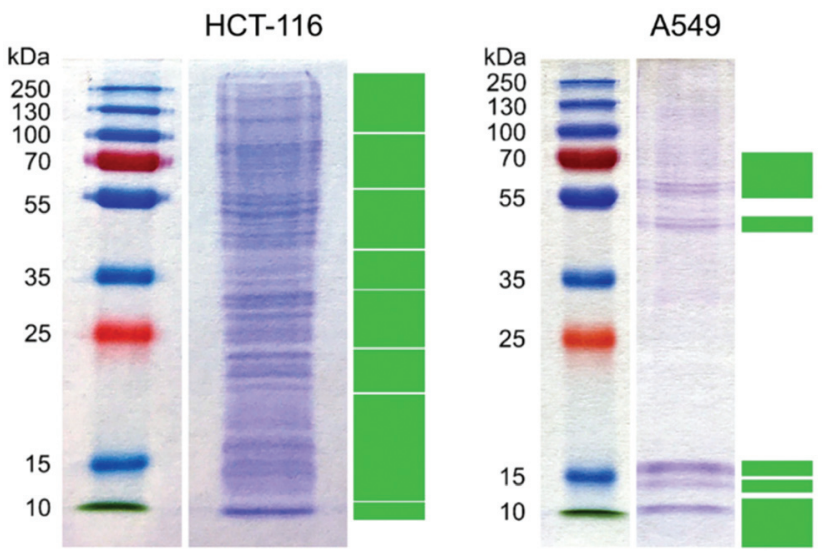

Fig. 1 Protein profile of the protein corona of gold nanoparticles internalized by HCT-116 and A549 cells in DMEM-FBS. Green rectangles mark the excised gel segments analyzed by HPLC-ESI-Q-TOF MS.

This provides direct information about the interacting proteins and informs about the possible cellular responses.

The electropherogram of the hard corona proteins extracted from HCT-116 and A549 cells, respectively, are shown in Fig. 1.

Despite some similarities in the identified hard corona proteins in Tables $\mathrm{S} 1$ and $\mathrm{S} 2, \dagger$ the two hard corona profiles are majorly different (Fig. 1 and Tables S1 and S2†). After the mass spectrometric analysis of the peptides extracted from the excised gel segments (marked with green solid rectangles in Fig. 1), 297 and 52 proteins were identified with MASCOT ${ }^{22}$ in the hard protein corona of gold nanoparticles in HCT-116 and in A549, respectively (see the lists of identified proteins in Tables $\mathrm{S} 1$ and $\mathrm{S} 2 \dagger$ ). Since the culture medium was supplemented with fetal bovine serum and the majority of the identified proteins was of human origin, it can be concluded that the analyzed hard protein corona was formed during and after the internalization of the nanoparticles, as also discussed in ref. 28. As seen in Fig. 1, only the most intense bands in the extracted hard corona proteins from A549 cells were excised for further mass spectrometric analysis, because proteins with low concentration increased the threshold of significance in the data sets.

The proteins listed in Tables S1 and $\mathrm{S} 2 \uparrow$ were first analyzed by DAVID. ${ }^{23,24}$ Twelve and four functional clusters were determined in the hard corona of gold nanoparticles in HCT-116 and A549, respectively, as shown in Table 1. As indicated in Table 1, bovine proteins were found in the hard corona proteome of both cell lines: four in that of HCT-116 and two in that of A549 (Table 1). Bovine hemoglobin subunit alpha and vitronectin were found in the respective protein coronas extracted from both cell lines (Tables S1 and S2 $\dagger$ ), and hemoglobin fetal subunit beta and a protein similar to bovine apolipoprotein B were additionally detected in the hard corona formed in HCT-116 (Table S1†). These proteins were present in the hard corona even after $24 \mathrm{~h}$ of incubation, which suggests their strong interaction with gold nanoparticles. In a complex protein solution, e.g., inside intracellular compartments, first, 
Table 1 Protein clusters based on the enrichment and functional assignment of hard corona proteins on gold nanoparticles recovered from HCT-116 and A549 cells. The functional assignment was done by DAVID $^{23,24}$

\begin{tabular}{l}
\hline Cluster \\
\hline Ribonucleoprotein-related \\
Translation initiation factor-related \\
Translation regulation-related \\
RNA-binding-related \\
RNA transport-related \\
Protein folding-related \\
Nucleosome-related \\
Helicase-related \\
GTP-binding-related \\
Endoplasmic reticulum-related \\
Mitochondrion-related \\
Porin-related \\
Glycolysis-related \\
Calcium ion binding-related \\
Unclassified \\
Bovine proteins \\
Total
\end{tabular}

$\begin{array}{ll}\text { HCT-116 } & \text { A549 } \\ 77 & - \\ 16 & - \\ 5 & - \\ 32 & - \\ 4 & - \\ 9 & - \\ 5 & 9 \\ 8 & - \\ 6 & - \\ 10 & - \\ 14 & - \\ 4 & - \\ - & 3 \\ - & 4 \\ 110 & 40 \\ 4 & 2 \\ 297 & 52\end{array}$

the most abundant proteins interact with the surface, which then gradually exchange to proteins with higher surface affinity because of the Vroman effect. ${ }^{29,30}$

The concentration of bovine serum albumin, the most abundant protein in fetal bovine serum-supplemented culture media, was below the detection limit, which suggests that bovine serum albumin only participates in weak interactions with the gold nanoparticles. This is in accordance with previous publications related to bovine serum albumin-gold nanoparticle interactions, ${ }^{13,31}$ that concluded only non-specific interactions between the exposed lysine residues of bovine serum albumin and the citrate ions adsorbed on the nanoparticle's surface.

The enrichment of some proteins with specific functions in the hard corona (see Tables S1 and S2 $\dagger$ ) allows for the discussion of their role in the cellular processing of the nanoparticles. A schematic representation of the assessed processing is shown in Scheme 1. As examples, annexins and ladinin indicate an interaction of the gold with cell membranes, ${ }^{32,33}$ as well as the interactions of nanoparticles with cilia (e.g., cilia- and flagella-associated protein 157 in HCT-116). Cytoplasmic actin is present in both protein corona profiles, which is responsible for the pit formation in the cell membrane during active uptake. ${ }^{34}$ The presence of clathrin light chain A and clathrin heavy chain 1, as well as AP-2 complex subunit alpha-1 in the hard corona suggests that gold nanoparticles can be internalized by clathrin-mediated endocytosis. $^{35,36}$ While these proteins are generally on the outer surface of vesicles, in multivesicular bodies and in compartments targeted for exocytosis, they can get in direct contact with the gold nanoparticles as well. ${ }^{10}$

After their internalization, gold nanoparticles induced different cellular response mechanisms in the two cell lines under study. In A549, the protein corona of gold nanoparticles contains vimentin, which is the highest scoring entry among

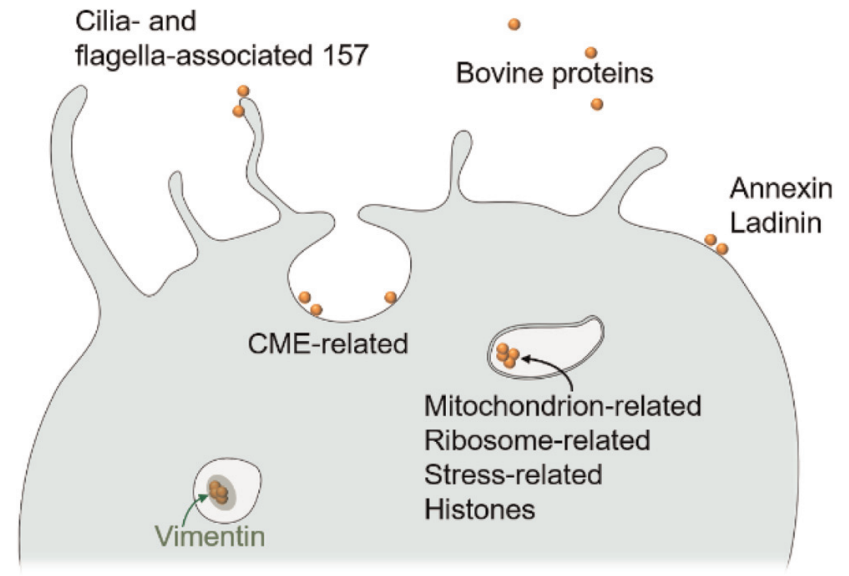

Scheme 1 Summary of the proteins involved in the processing of gold nanoparticles by cells as indicated by the proteomics data of the extracted hard protein corona. After uptake by e.g., clathrin-mediated endocytosis (CME), nanoparticles are processed via the endolysosomal pathway that may direct them to single-membrane endosomes or double-membrane autophagosomes.

the heavier proteins based on the algorithms in MASCOT $^{22}$ (Table $\mathrm{S} 2 \dagger$ ). Vimentin is involved in the formation of aggresomes, which are intracellular bodies formed by the grouping of aggregated, denatured proteins that are meant to be processed further. ${ }^{37}$

In contrast, the corona extracted from HCT-116 is exempt from vimentin as it is not expressed in this cell line ${ }^{38}$ and shows very distinct properties and a much higher protein diversity. In total, 77 different ribosome-related proteins and 14 mitochondrial proteins are present. Since gold nanoparticles are most often stored in different intracellular compartments, they cannot interact with ribosomes and mitochondria in the cytosol, but only inside endolysosomal organelles, e.g., autophagolysosomes. These compartments are crucial for the processing of damaged or unwanted intracellular materials, and they help in their elimination in the form of extracellular vesicles. ${ }^{39,40}$ In comparison to the DAVID analysis of the hard corona proteome of A549, suggesting complete absence of ribosomal and mitochondrial proteins, STRING ${ }^{25}$ analysis, based solely on protein functions but not on enrichment, classified ten proteins related to ribosomes and zero to mitochondria.

Both cell lines associate gold nanoparticles with histones (Tables S1 and S2 $\dagger$ ), which are the primary component in the bands corresponding to 10-15 kDa proteins in Fig. 1. Histones can adsorb on the surface of gold nanoparticles in extracellular vesicles or vesicles targeted to be excreted, most frequently in apoptotic bodies. ${ }^{41}$ Apoptotic bodies contain intact organelles with cytoplasmic matter and, in some cases, nuclear fragments. In such extracellular vesicles, gold nanoparticles can interact with histones, ribosomes, and mitochondria as well. Moreover, 23 and 15 stress-related, and 41 and 14 apoptosisrelated proteins were also identified by $\mathrm{STRING}^{25}$ in the hard coronas extracted from HCT-116 and A549 cells, respectively, 


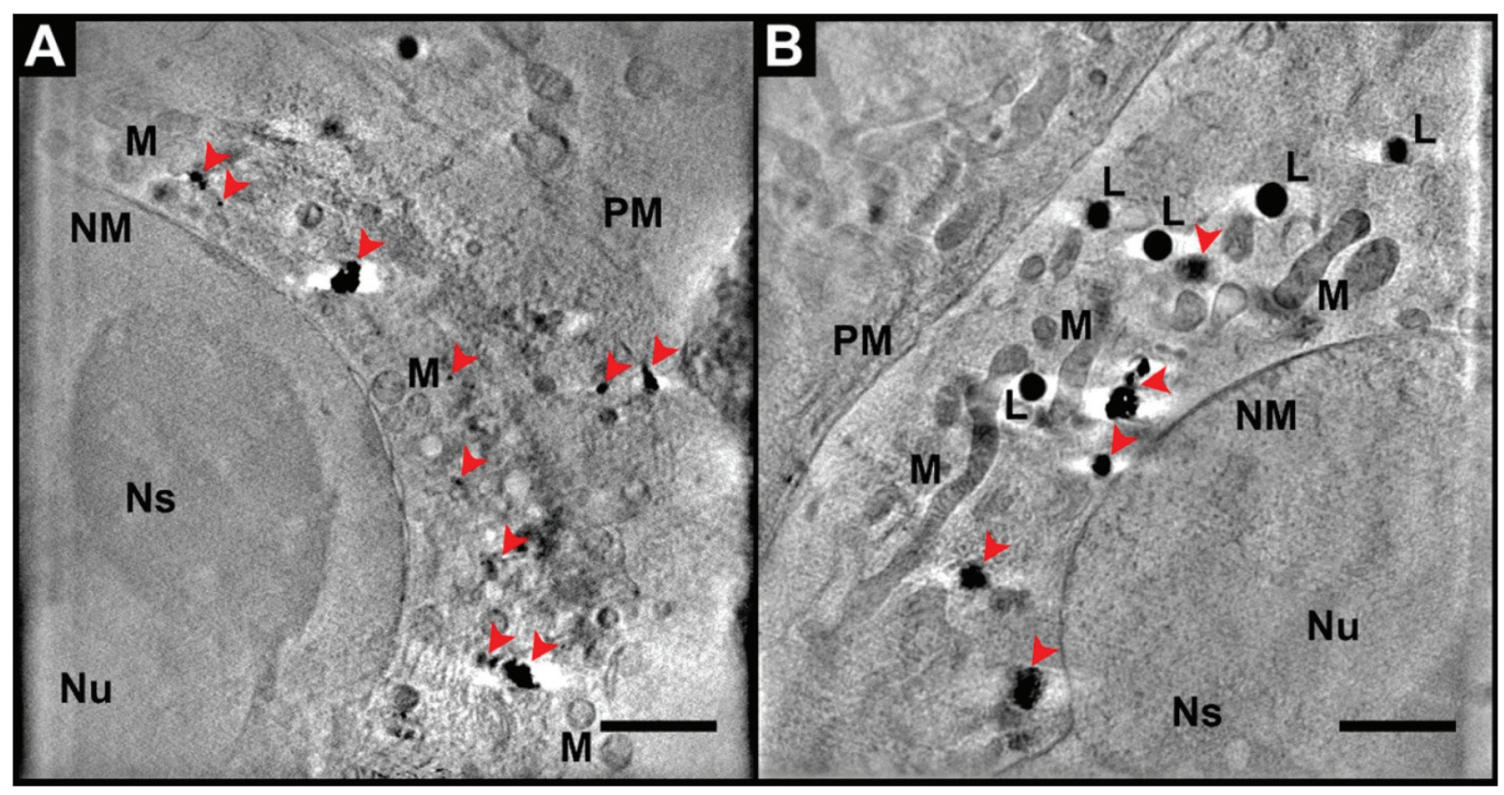

Fig. 2 Tomographic slices of HCT-116 cells grown in (A) DMEM-FBS and (B) McCoy-FBS incubated with non-aggregated gold nanoparticles. M: mitochondrion, L: liposome, PM: plasma membrane, NM: nuclear membrane, Nu: nucleus, Ns: nucleolus. Gold nanostructures are marked with red arrowheads. Scale bar: $2 \mu \mathrm{m}$

which further supports that apoptotic processes were activated in the presence of gold nanoparticles.

\section{Ultrastructural changes in the course of nanoparticle uptake and processing}

To study potential effects of the culture medium composition on nanoparticle uptake, processing, and interaction of the proteins in the hard corona, both cell lines were cultured in DMEM-FBS and in McCoy's modified 5A medium supplemented with $10 \%$ fetal bovine serum (McCoy-FBS). While McCoy-FBS is suggested by the distributor of HCT-116 for cultivation, DMEM-FBS is widely used in experiments with this cell line, ${ }^{42-44}$ which could affect the viability of cells in case of incubation with nanoparticles. ${ }^{45}$ As a possible result of McCoyFBS being more supplemented than DMEM-FBS, ${ }^{46}$ HCT-116 cells grew slower in DMEM-FBS than in McCoy-FBS as observed under a light microscope (data not shown). In contrast, the growth rates of A549 cells in the two culture media were comparable to each other. The gold nanoparticles were added to each culture medium in non-aggregated form and as nanoaggregates, respectively, to study the potential influence of larger size and different surface properties due to the aggregation by $\mathrm{NaCl}$ on the processing. As a cellular response to the internalization of gold nanoparticles and their intracellular accumulation, ultrastructural changes are expected to be induced in the course of nanoparticle processing. Cryo soft X-ray nanotomography (cryo-SXT) experiments were performed, which yield three-dimensional ultrastructural information about organelles and the internalized gold nanoparticles.
Tomographic slices of HCT-116 cells incubated for $24 \mathrm{~h}$ with non-aggregated gold nanoparticles in DMEM-FBS and in McCoy-FBS, respectively, are shown in Fig. 2. The ultrastructural features of A549 cells after their incubation with nonaggregated gold nanoparticles are shown in Fig. S2A and S3A. $\dagger$ In both media, the gold nanoparticles form aggregates inside the cells, in agreement with processing of these nanoparticles by other cell lines. ${ }^{11,12}$ In accordance with the proteomics and mass spectrometric results of proteins related specifically to clathrin-mediated endocytosis in the hard corona proteome (Table S1†) that results in only a few nanoparticles in the same vesicle, we conclude that the aggregate formation occurred during processing inside the cells.

In HCT-116, the increased number of small, round mitochondria suggests mitochondrial fission (Fig. 2A), and the dilated perinuclear cisternae (Fig. 2A) indicate that apoptosis could have started in the cells ${ }^{47-50}$ when cultured in DMEM-FBS and after uptake of the non-aggregated gold nanoparticles. In contrast, when the cells were grown in McCoy-FBS and incubated with the gold nanoparticles (Fig. 2B), longer mitochondria and intact nuclear membrane were observed. This suggests that the use of DMEM-FBS may promote cellular stress by insufficient nutrition, e.g., a lack of biotin, vitamin B12, or ascorbic acid or low choline concentration, ${ }^{46}$ which specifically has been shown to promote apoptosis. ${ }^{51}$ Although the mitochondrial cristae are well contoured, suggesting that these organelles are intact here (Fig. 2A), it is known that gold nanoparticles can trigger mitochondrial damage, which results in mitochondrial fission or apoptosis. ${ }^{40}$ The tomograms of HCT-116 in DMEM-FBS suggest that the cellular and organelle damage upon gold nanoparticle uptake is more pro- 
minent at less optimal culture conditions, possibly because of a lower efficiency of the processes that promote survival. Contrary to HCT-116, A549 cells appeared healthy in both cell culture media. Nevertheless, their mitochondria are slightly more abundant when the cells were grown in DMEM-FBS (Fig. S2A $\dagger$ ) than in McCoy-FBS (Fig. S3A $\dagger$ ).

In opposition to the incubation with individual nanoparticles, HCT-116 cells show signs of stress in both culture media upon incubation with nanoparticles that were aggregated by the addition of sodium chloride prior to uptake (Fig. S4†). Mitochondrial fission (not shown) or hyperfusion (Fig. S4A $\dagger$ ) can be observed in the whole tomogram due to mitochondrial damage and higher energy demand, ${ }^{49,52}$ respectively. In the nucleus, chromatin granules form (Fig. S4 $\uparrow$ ), and large vacuoles with bright lumen appear in the cytoplasm (Fig. S4B $\dagger$ ). The virtual segmentation of the whole tomogram (Fig. 3, corresponding to the section shown in Fig. S4B $\dagger$ ) clearly shows the high number of chromatin granules (Fig. 3, blue volumes) that are distributed over the entire nucleus, and the high intracellular volume occupied by the cytoplasmic vacuoles (Fig. 3, red volumes). This indicates that the excessive endocytosis of the pre-aggregated gold nanoparticles induces cellular damage,$^{53-55}$ that can ultimately lead to apoptosis and autophagy. ${ }^{40}$ The large cytoplasmic vacuoles can also originate from the dilation of the endoplasmic reticula $^{53}$ or other processes related to the water permeation of the cell membrane. ${ }^{54}$ The high density of the chromatin granules, resulting in higher absorption, ${ }^{56}$ suggests that they must be tightly packed, as a possible result of apoptosisinduced chromatin condensation, ${ }^{55}$ and different from typical, naturally abundant, heterochromatin patches (compare also Fig. S4† with Fig. 2).

When A549 cells are incubated with pre-aggregated gold nanoparticles, similar signs of cellular injury and stress are observed (Fig. S2B and S3B $\dagger$ ). They include the altered mitochondrial morphologies as in the HCT-116 cells, leading us to infer on changes in energy production and demand of the

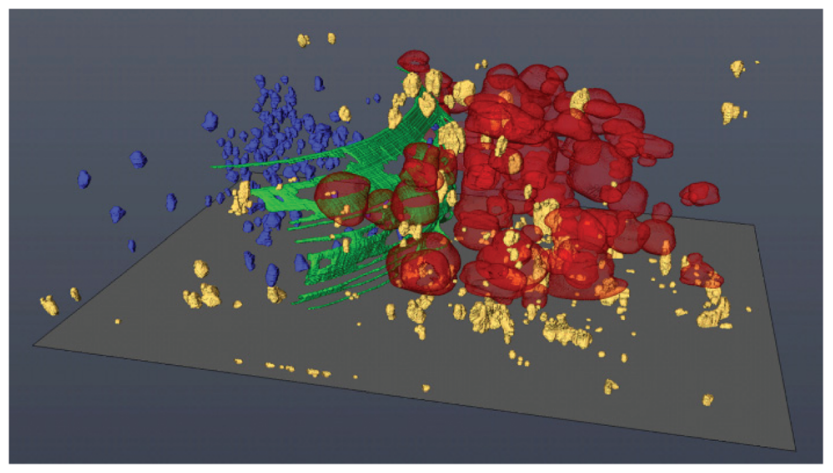

Fig. 3 Segmentation of the tomogram of an HCT-116 cell, incubated in McCoy-FBS, with pre-aggregated gold nanoparticles (also represented by a tomographic slice in Fig. S4B $\uparrow$ ). Red volumes: vacuoles, yellow volumes: gold nanoparticles, green mesh: nuclear membrane, blue volumes: chromatin granules. cells. Damage and cell death are also revealed by chromatin granules formed in the nucleus (Fig. S3B $\dagger$ ) and by the disruption of the nuclear membrane (Fig. S2B $\dagger$ ). The formation of extracellular vesicles can be observed in Fig. S3B. $\dagger$ Relating this particular observation from cryo-SXT to the mass spectrometric results, e.g., the presence of caspase-14, histones, and heat-shock proteins (Table S2 $\dagger$ ), it is most likely that they are apoptotic bodies.

The virtual segmentation of tomograms permits an analysis of the amount of nanoparticles internalized by the different cell lines and under different conditions, and of the morphology of the nanoaggregates during the processing by the cells. ${ }^{11,18}$ The intracellular gold nanoparticle aggregates in both cell lines were highlighted by segmentation (Fig. 4), and their size was analyzed.

The average intracellular aggregate size increased when the cells were incubated with the pre-aggregated gold nanoparticles (Fig. 4B, D, F and $\mathrm{H}$ ) compared to the incubation with non-aggregated gold nanoparticles (Fig. 4A, C, E and G). After incubation with individual gold nanoparticles, the rendered structures, corresponding to the intracellular nanoaggregates, have a more homogeneous size (indicated by a lower standard deviation) in the A549 cells (Fig. 4E and G with a standard deviation of \pm 129 and \pm 190 nanoparticles per aggregate, respectively) than in HCT-116 cells (Fig. 4A and C with a standard deviation of \pm 445 and \pm 228 nanoparticles per aggregate, respectively). This shows the important influence of the intracellular processing of the nanoparticles, which in the case of uptake of individual nanoparticles is specific for each cell line.

From the number and size of the intracellular nanoparticle aggregates and the size of the pristine nanoparticles, the number of nanoparticles internalized by the cells in the different culture media can be estimated (Table S3†). Regardless of the number of internalized aggregates, in both cell culture media and both cell lines, the total number of internalized nanoparticles is higher in the case of incubation with pre-aggregated gold nanoparticles than with non-aggregated nanoparticles (compare columns in Table S3†). This overall increase is the result of the higher number of simultaneously internalized particles in the form of aggregates compared to the case of single nanoparticle uptake.

We explain the higher intracellular gold nanoparticle number in HCT-116 when cultured in DMEM-FBS (Table S3, $\dagger$ compare first two rows) by the sub-optimal nutrition of the cells in this medium, possibly leading to the recognition of the nanoaggregates as a source of nutrients by the cells. The pre-formed gold nanoaggregates are covered with a primary protein corona formed in the culture medium when they reach the cell membrane, and it is possible that the cell internalizes them more readily than in McCoy-FBS, that is, under optimum nutrient conditions. As discussed above, also in A549 cells the signs of cellular stress become more prominent in the case of incubation with pre-aggregated gold nanoparticles (Fig. S2 and $\mathrm{S} 3 \dagger)$. While the amount of nanoparticles contained in intracellular aggregates after incubation with individual gold nano- 


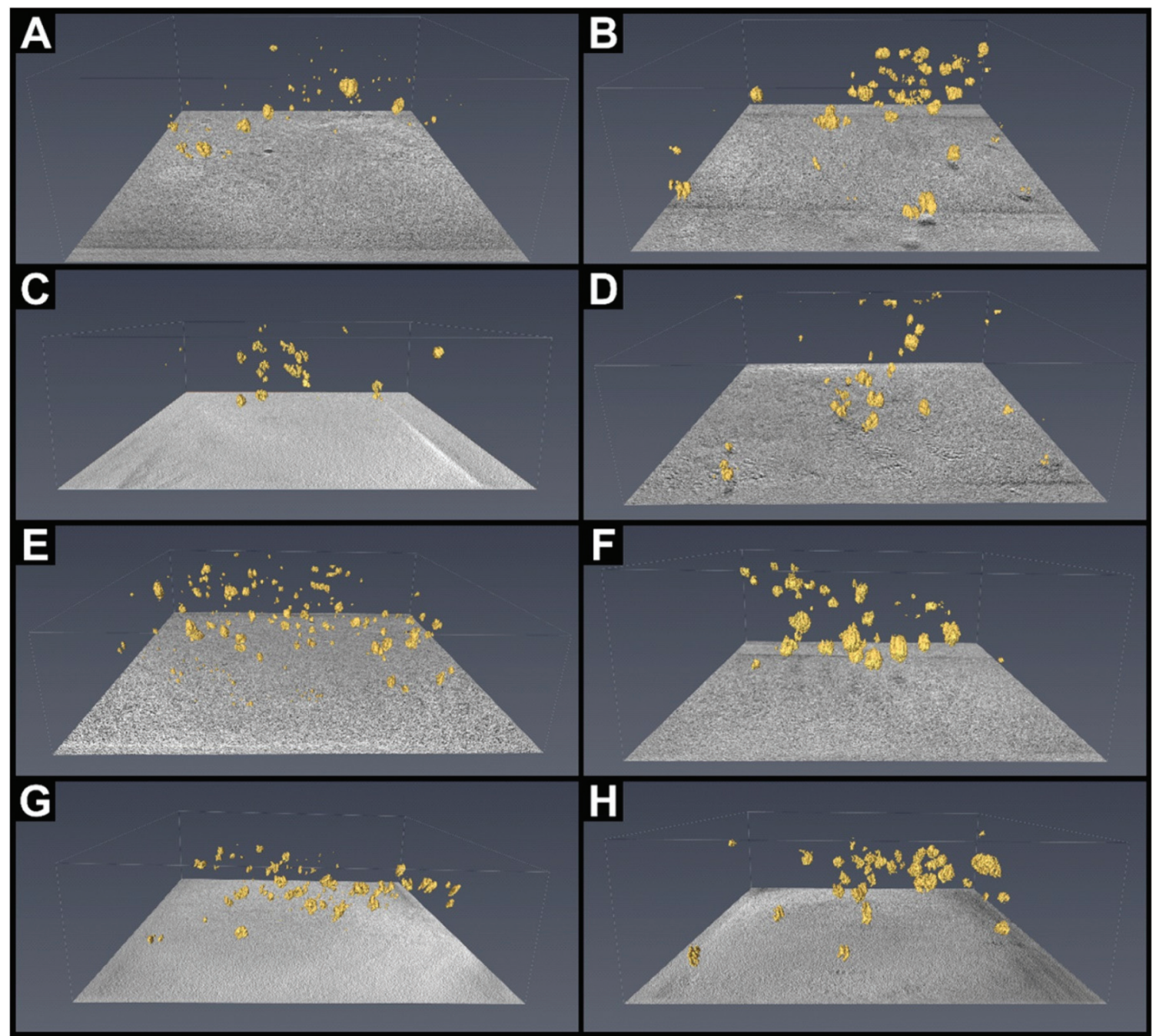

Fig. 4 Segmentation reveals gold nanoparticle aggregates in the tomograms (Fig. 2 and Fig. S2-4†): of (A) an HCT-116 cell grown in DMEM-FBS with non-aggregated (cf. Fig. 2A) and (B) pre-aggregated gold nanoparticles (cf. Fig. S4A $\dagger$ ), (C) an HCT-116 cell grown in McCoy-FBS incubated with non-aggregated (cf. Fig. 2B) and (D) pre-aggregated gold nanoparticles (cf. Fig. S4B †). (E) A A549 cell grown in DMEM-FBS incubated with nonaggregated (cf. Fig. S2A $\dagger$ ) and (F) pre-aggregated gold nanoparticles (cf. Fig. S2B $\dagger$ ), respectively and (G) a A549 cell grown in McCoy-FBS incubated with non-aggregated (cf. Fig. S3A $\dagger$ ) and $(\mathrm{H})$ pre-aggregated gold nanoparticles (Fig. S3B $\dagger$ ), respectively.

particles is similar in both culture media, the number of gold nanoparticles taken up in the form of nanoaggregates increased in DMEM-FBS by over 37\% compared to incubation in McCoys-FBS (Table S3, $\dagger$ compare bottom two rows). The higher numbers of internalized gold nanoparticles, together with the observed stress- and cellular injury-related changes in cellular ultrastructure, (Fig. S2B and S3B $\dagger$ ), lead to the conclusion that the nanoparticle uptake is higher under higher cellular stress.

In summary, the cryo-SXT data clearly show that the uptake of gold nanoparticles can induce cellular stress and injury on the single-cell level. The proteomics and mass spectrometric results in Tables $\mathrm{S} 1$ and $\mathrm{S} 2, \dagger$ as well as the XTT results shown in Fig. S5 $\dagger$ are in agreement with the conclusions of the cryoSXT data. The extent of the ultrastructural changes connected to cellular injury and cell death depends both on the cell line and on the chosen incubation conditions. Specifically, the cellular stress as well as nanoparticle uptake are enhanced by depleted culture medium, as in the case of HCT-116 in DMEM-FBS. Furthermore, uptake of the nanoparticles in the form of sodium chloride-induced nanoaggregates enhances the effect in both cell lines. This observation is in agreement with the role of the hard corona as interface with its specific physicochemical properties between the surface of the pristine gold nanoparticles and the processing by the cellular structures.

\section{The processing of nanoparticles and their accumulation are influenced directly by the hard corona composition}

While the hard corona composition at a certain stage of cellular nanoparticle processing depends on the preceding processing pathways, it also determines the subsequent fate of the 
internalized nanoparticle. The average number of nanoparticles per aggregate was nearly constant in HCT-116, regardless of whether the particles were administered as single or pre-aggregated gold nanoparticles (Table S3†). This indicates that the HCT-116 cells process the nanoparticles and their aggregates in a way that yields similar particle numbers per intracellular aggregate. In contrast, in A549, more substantial differences were found: the average number of particles per aggregate increased in both media when the cells were incubated with pre-aggregated nanoparticles instead of individual particles (Table S3†). This could suggest that the internalized, pre-formed aggregates are preserved after uptake, and that the individual nanoparticles become part of aggregates that form during endolysosomal processing. Furthermore, while the total number of aggregate structures increased in HCT-116 when the internalized particles were pre-aggregated (Fig. 4AD), in A549, their number decreased (Fig. 4E-H).

The difference between the nanoparticle processing of the two cell lines can be explained by relating the aggregate morphological properties obtained from cryo-SXT experiments to the results of the proteomic analysis of the hard corona discussed in the beginning. As observed by mass spectrometry, one of the most abundant proteins in the hard corona extracted from A549 is vimentin (Table S2 $\dagger$ ), which plays a key role in intracellular aggresome formation. ${ }^{37}$ As mentioned above, aggresomes are the deposit sites of aggregated or misfolded proteins regulated by the cell. In line with the rationale that the proteins forming the corona are what the cell "sees", ${ }^{2}$ these results indicate that the A549 cells might see the nanoparticle corona as a form of aggregated proteins. The formation of a vimentin cage around the gold nanoparticles was previously shown to reduce oxidative stress. ${ }^{57}$ This can explain why the A549 cells, due to high abundance of vimentin in the hard corona are more robust and show less stress-related changes upon the uptake of gold nanoparticles than HCT-116 cells do.

HCT-116 cells process the nanoparticles and their aggregates based on different mechanisms, as also supported by a different hard corona proteome (Table S1†). Based on the presence of proteins with a role in clathrin-mediated endocytosis in the hard corona, this pathway is a possible uptake mechanism for the internalization of the gold nanoparticles by HCT-116. However, clathrin-mediated endocytosis only explains the uptake of the single particles or of small aggregates corresponding to 2-6 particles. ${ }^{58-60}$ For uptake of larger structures, such as the pre-formed aggregates hereby HCT-116, another mechanism has to be activated. It has been shown that HCT-116 cells readily combine clathrin-mediated endocytosis with macropinocytosis during the uptake of nanoparticles. ${ }^{61}$ The tomographic segmentation data show that in A549 the total number of intracellular aggregates decreases upon incubation with pre-aggregated gold nanoparticles compared to HCT-116, which suggests that such an uptake via macropinocytosis must be less abundant in A549 cell. This is in agreement with previous discussions that macropinocytosis is not a prevalent uptake mechanism by A549 cells. ${ }^{62}$

\section{Interactions at the hard corona-gold interface}

To reveal the interactions of the hard corona proteins identified by mass spectrometry and the surface of the gold nanoparticles, SERS spectra were measured from the intracellular nanoaggregates in living cells in mapping experiments. Acknowledging the typical variations in relative band intensities and signal fluctuation inherent to a SERS experiment, a total of $\sim 5300$ individual spectra that yielded a signal, i.e., from gold nanoparticles inside the cells, were analyzed regarding the occurrence of bands. This enables an identification of those molecular groups that are in direct proximity of the gold nanoparticle surface and a discussion of spectral patterns based on co-occurrences, independent of relative band intensities, that can be compared for different cell lines, incubation media, and nanoparticles/nanoaggregates (Fig. 5, band assignments in Table $\mathrm{S} 4 \dagger$ ). The spectral patterns differ based on

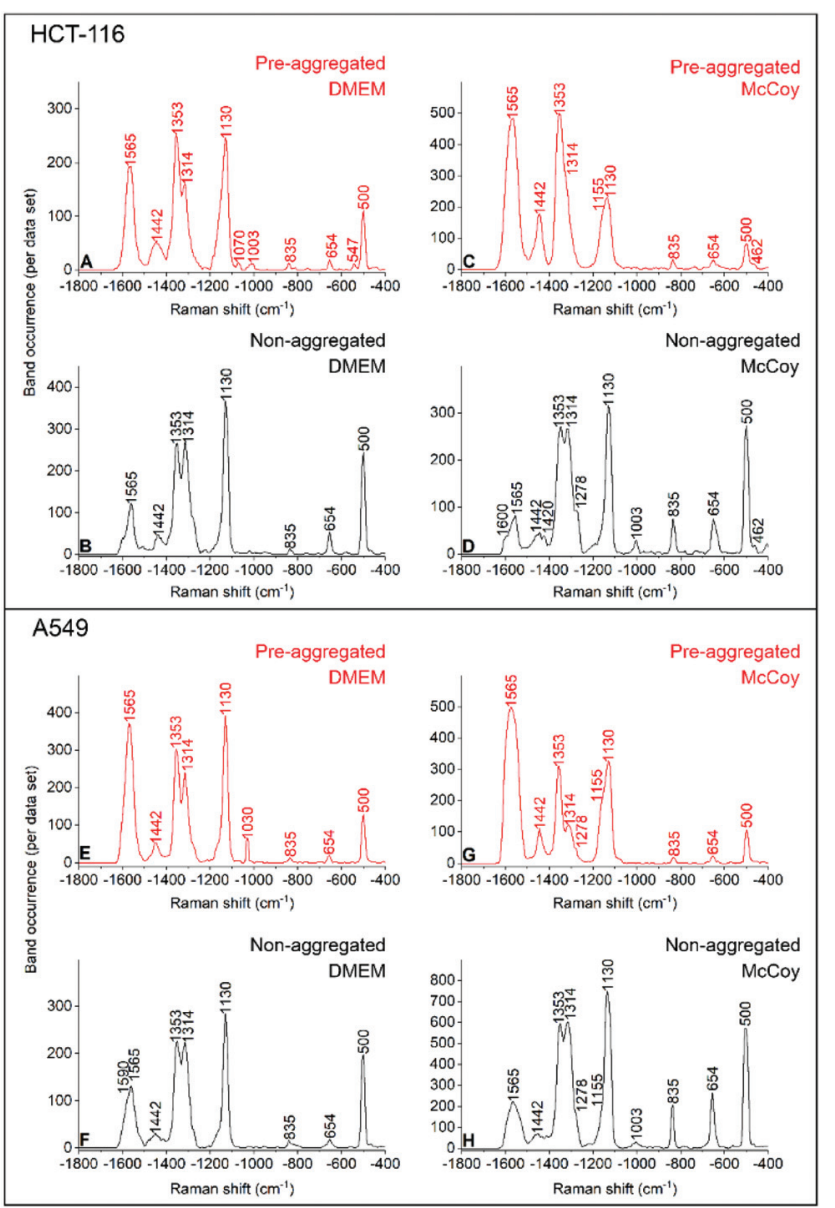

Fig. 5 Band occurrence determined from the respective SERS data sets of (A and B) HCT-116 cells grown in DMEM-FBS and (C and D) McCoy$F B S$, and of $A 549$ cells grown in DMEM-FBS ( $E$ and F) and McCoy-FBS (G and $H$ ), after incubation with gold nanoaggregates ( $A, C, E$ and $G$ ) and individual gold nanoparticles (B, D, F and $\mathrm{H}$ ). Each curve represents SERS spectra from five individual cells per incubation condition, yielding 627 (A), 661 (B), 705 (C), 402 (D), 823 (E), 602 (F), $604(G)$, and $909(H)$, spectra, respectively, per data set. The original spectra were recorded at $785 \mathrm{~nm}$ excitation. 
whether the cells were incubated with non-aggregated or preaggregated gold nanoparticles (compare red and black traces in Fig. 5), but they are independent of the cell line or the chosen culture medium. The band at $1130 \mathrm{~cm}^{-1}$, assigned to the $\mathrm{C}-\mathrm{C}$ and $\mathrm{C}-\mathrm{N}$ stretching vibrational contribution of the peptide backbone (assignments in Table $\mathrm{S} 4 \dagger$ ) is present in the majority of the spectra (Fig. 5). It is also prominent in the average spectra calculated from each of the data sets (Fig. S6†). In all data collected from the cells incubated with the nanoparticles that were pre-aggregated with sodium chloride, the occurrence of this band compared to signals around $1565 \mathrm{~cm}^{-1}$ that are assigned to tryptophan, tyrosine, amide II, and $\mathrm{COO}^{-}$is lower than in the data collected with non-aggregated nanoparticles (Fig. 5, compare red and black curves in each panel).

In the corresponding average spectra from the samples with pre-aggregated nanoparticles this reflects in a lower band ratio (Fig. S6, $\dagger$ red spectra). At the same time, the signal occurrence (or average band intensity) at $1353 \mathrm{~cm}^{-1}$, from another vibrational mode of tryptophan compared to that of an amide III mode at $1314 \mathrm{~cm}^{-1}$ (Table $\mathrm{S} 4 \dagger$ ) is higher in the average spectra of the cells with pre-aggregated nanoparticles, which indicates the promotion of hydrophobic interactions between proteins and the nanoparticle surface. The frequent occurrence of signals attributed to the non-polar residues on the pre-aggregated nanoparticles is accompanied by a lower contribution of the signal at $500 \mathrm{~cm}^{-1}$ assigned to the disulfide S-S stretching compared to that at $654 \mathrm{~cm}^{-1}$ of cysteine $\mathrm{C}-\mathrm{S}$ stretching vibrations. The lower contribution from $\mathrm{S}-\mathrm{S}$ indicates that the secondary structure of proteins composing the corona must be compromised in this case, as disulfide bonds stabilize the protein folding.

The nanoparticle-side chain interactions reveal themselves in more detail in the following co-occurrences of abundant spectral bands. The band at $1565 \mathrm{~cm}^{-1}$, assigned to vibrations of tryptophan, tyrosine, amide II, and $\mathrm{COO}^{-}$often appears together with the $1353 \mathrm{~cm}^{-1}$ band assigned to tryptophan vibrations in cells (Table S4 $\dagger$ ). Although in principle an assignment to other biomolecules than proteins is possible, we consider the contribution of guanine to the band at $1353 \mathrm{~cm}^{-1}$ in these data unlikely, based on its co-occurrence with other typical protein modes. With the non-aggregated nanoparticles (Fig. 5, black traces), the $1353 \mathrm{~cm}^{-1}$ tryptophan signal is mostly independent of the $1314 \mathrm{~cm}^{-1}$ amide III band component and of the $500 \mathrm{~cm}^{-1} \mathrm{~S}-\mathrm{S}$ stretching signal, respectively. The co-occurrence of the amide III and disulfide bands indicates intact structural elements in the secondary structure of the proteins composing the corona on the non-aggregated nanoparticles (Fig. 5, black traces). Also, in some cases, bands attributed to aromatic side-chain vibrations, e.g., at $1565 \mathrm{~cm}^{-1}$ and $1353 \mathrm{~cm}^{-1}$, were found to co-occur with the S-S stretching vibration, in agreement with the concentration of aromatic residues around disulfides in proteins. ${ }^{63}$ When present, the band at $654 \mathrm{~cm}^{-1}$ cystine $\mathrm{C}-\mathrm{S}$ stretching appears nearly exclusively with an $\mathrm{S}-\mathrm{S}$ stretching vibration, indicating the presence of intact disulfide bonds. The co-occurrence of these bands suggests the interaction of the gold nanoparticle surface with one of the sulfur atoms in a disulfide bridge, which can take place via a nonbonding electron pair of the sulfur atom, as proposed recently. ${ }^{64}$

The stronger contribution by signals of nonpolar group vibrations in the cells incubated with pre-aggregated particles compared to those incubated with non-aggregated nanoparticles must be discussed in the context of the $\zeta$-potential of the nanoparticles as most important determinant of the interfacial properties of the nanoparticles. As has been discussed previously in other work, the decrease of the absolute $\zeta$-potential of the gold nanoparticles when sodium chloride is added $^{65,66}$ to achieve pre-aggregation in our experiments leads to a gold surface with lower affinity for electrostatic interactions. ${ }^{67}$ Since the interaction between proteins and nanoparticles is the combination of electrostatic and hydrophobic interactions, together with other effects, ${ }^{67}$ weakening of the electrostatic component causes increased hydrophobicity of the gold nanoparticles. ${ }^{65}$ This promotes the hydrophobic component of protein-nanoparticle interactions. Therefore, the low absolute $\zeta$-potential of the pre-aggregated gold nanoparticles affects the formation of the primary corona in the moment of their addition to the culture medium. Such change on the potential results in protein adsorption based more on hydrophobic than electrostatic interactions, as observed in the SERS data of cells incubated with pre-aggregated gold nanoparticles. In contrast, when the nanoparticles are not preaggregated, the interactions in the rapidly formed primary protein corona rely more on electrostatic interactions. At the same time, the proteins partially shield the surface of the nanoparticles from the higher ion concentration of the culture medium or the intracellular environment that could potentially change their surface properties rendering them more prone to interact with hydrophobic side chains. Since the protein corona forms a multi-layer coverage on the gold nanoparticles, the exchange of the proteins directly interacting with the nanoparticles occurs in a protected environment that at least partially prevents the exposure of the nanoparticle surface to the environment with higher ionic strength.

In the culture conditions that led to cellular stress and damage, specifically in the HCT-116 cells grown in DMEM-FBS and incubated with the pre-aggregated nanoparticles, SERS spectra also give molecular evidence of the changes that were observed at the level of subcellular ultrastructure (Fig. S4A $\dagger$ ). The vibration at $1070 \mathrm{~cm}^{-1}$ appears together with the bands at $1110 \mathrm{~cm}^{-1}$ assigned to the $\mathrm{C}-\mathrm{C}$ stretching of carbohydrates, lipids, and proteins, ${ }^{68} 1190 \mathrm{~cm}^{-1}$ assigned to cytosine, guanine, adenine, and antisymmetric phosphate vibration, ${ }^{68}$ and $1470 \mathrm{~cm}^{-1}$ assigned to $\mathrm{C}=\mathrm{N}$ deformation of nucleobases and lipids. ${ }^{68}$ Even though the band at $1470 \mathrm{~cm}^{-1}$ can be assigned to the $\mathrm{C}-\mathrm{H}$ deformations of proteins as well, ${ }^{69}$ the cooccurrence of these bands, not observed in the other data sets, suggests the interaction of gold nanoparticles with nucleic acids, in agreement with the induced cell death process, and also by the disintegration of mitochondria and ribosomes, and by nuclear fragmentation (Fig. S4A and Table S1†). Based on 
the mass spectrometric, cryo-SXT, and cell viability results, the stronger contribution of nucleic acids to the SERS signal in this sample compared to the others is the result of the significant cellular damage caused by the internalized gold nanoparticle aggregates.

\section{Conclusions}

Using the two cell lines, HCT-116 and A549, incubated with gold nanoparticles that were applied as individual particles and as nanoaggregates, data on protein corona composition from proteomics and mass spectrometry, on ultrastructural changes in the cells from cryo-SXT, and on the uptake, processing, and interfacial interactions of the nanostructures studied by SERS were combined. Furthermore, a detailed characterization of the protein interaction and composition at the immediate surface of the gold nanostructures was undertaken. Thereby, the composition and structure of the corona proteins were directly linked to the physicochemical properties of the gold nanostructures on the one hand, and associated with the nanoparticle processing on the other hand, underpinning the important mediator function of the hard protein corona. In summary, the results show that not only are the processing of nanoparticles and their accumulation directly influenced by the hard corona composition, but that the latter is determined by the interactions at the hard corona-gold interface. The different perspectives on the protein-nanoparticle interactions and their effects were combined by using MSbased proteomics, SERS, and cryo-SXT.

The hard corona contains proteins that participate in essential events in the cellular processing of the gold nanoparticles, in particular during their interaction with the outer surface of the cell and their clathrin-mediated endocytosis. The protein composition also indicates the role of the hard corona in induced cellular response mechanisms that include protein elimination and cell death. It was found that several bovine proteins deriving from the culture medium remain in the hard protein corona, which suggests their high affinity to the gold nanoparticle surface. Interestingly, the most abundant serum protein, bovine serum albumin, was not found in the hard protein corona above the significance threshold, which suggests its low affinity to the gold nanoparticle surface compared to other proteins.

The number, shape, and integrity of the cellular compartments, as determined from the rendering of soft-X-ray nanotomograms, suggest that the internalized gold nanoparticles induce cellular stress in both HCT-116 and A549 cells, albeit to varied extent depending on the cell culture and incubation conditions. Cells from cell line A549 were more robust and showed severe cellular damage only when the nanoparticles were internalized as nanoaggregates. This robustness is supported by the presence of the protein vimentin in the hard corona proteome extracted from A549 cells, known to alleviate oxidative stress. ${ }^{57}$ Together with the proteomics data, also the number of intracellular nanoparticles and of their aggregates obtained from the quantitative analysis of the segmented tomograms in both cell lines, the results clearly indicate different uptake mechanisms and intracellular nanoparticle processing.

While significant ultrastructural differences were found, the interactions at the immediate nanoparticle surface were independent of the cell line or the choice of culture medium. The SERS data showed that when the absolute $\zeta$-potential is decreased, as was the case in the administered gold nanoaggregates, the surface interactions rely more on hydrophobic than on electrostatic effects, which has implications for the interaction and structure of the hard corona proteins, and for the corona composition. The band occurrences and co-occurrences revealed that in the case of each incubation condition, the secondary structure of the adsorbed proteins remains at least partially intact. The stronger contribution of the nonpolar group vibrations on the nanoaggregates, represented, e.g., by the contributions from tryptophan support the preference of hydrophobic interactions over electrostatic interactions. The SERS data also corroborate the signs of cellular damage indicated by the XRT results in HCT-116 cells incubated with pre-aggregated gold nanoparticles in DMEM-FBS, as evidenced by increased spectral contributions of nucleic acids. Even though the experiments revealed major differences in the protein corona composition, nanoparticle uptake mechanism, and in the level of induced cellular stress, the SERS results clearly indicate that the governing nanoparticleprotein interactions depend mainly on the surface properties of the nanoparticles.

\section{Conflicts of interest}

The authors declare no conflicts of interest.

\section{Acknowledgements}

The authors thank HZB for the allocated beam time and experimental support at BESSY II, and for the help of Alaa Adawy Mohamed Hassan and Silvia Candás Zapico (Universidad de Oviedo, Oviedo, Spain) with transmission electron microscopy. G. P. S. and V. Z. gratefully acknowledge funding by a fellowship of the School of Analytical Sciences Adlershof (DFG GSC 1013) and J. K. by ERC grant no. 259432. M. M. B. and J. B. acknowledge the funding from CTQ2017-83569-C2-1-R, RTI2018-094605-BI00 and FC-GRUPIN-IDI/2018/000242.

\section{References}

1 T. Cedervall, I. Lynch, S. Lindman, T. Berggard, E. Thulin, H. Nilsson, K. A. Dawson and S. Linse, Proc. Natl. Acad. Sci. U. S. A., 2007, 104, 2050-2055.

2 D. Walczyk, F. B. Bombelli, M. P. Monopoli, I. Lynch and K. A. Dawson, J. Am. Chem. Soc., 2010, 132, 5761-5768. 
3 P. Aggarwal, J. B. Hall, C. B. McLeland, M. A. Dobrovolskaia and S. E. McNeil, Adv. Drug Delivery Rev., 2009, 61, 428437.

4 N. Fernandez-Iglesias and J. Bettmer, Nanoscale, 2015, 7, 14324-14331.

5 M. A. Dobrovolskaia, B. W. Neun, S. Man, X. Ye, M. Hansen, A. K. Patri, R. M. Crist and S. E. McNeil, Nanomedicine, 2014, 10, 1453-1463.

6 D. Docter, U. Distler, W. Storck, J. Kuharev, D. Wunsch, A. Hahlbrock, S. K. Knauer, S. Tenzer and R. H. Stauber, Nat. Protoc., 2014, 9, 2030-2044.

7 A. Cox, P. Andreozzi, R. Dal Magro, F. Fiordaliso, A. Corbelli, L. Talamini, C. Chinello, F. Raimondo, F. Magni, M. Tringali, S. Krol, P. Jacob Silva, F. Stellacci, M. Masserini and F. Re, ACS Nano, 2018, 12, 7292-7300.

8 F. Bertoli, D. Garry, M. P. Monopoli, A. Salvati and K. A. Dawson, ACS Nano, 2016, 10, 10471-10479.

9 Y. Yue, R. Behra, L. Sigg, M. J. F. Suter, S. Pillai and K. Schirmer, Environ. Sci.: Nano, 2016, 3, 1174-1185.

10 G. P. Szekeres, N. Fernandez-Iglesias, J. Kneipp, M. MontesBayon and J. Bettmer, J. Proteomics, 2020, 212, 103582.

11 D. Drescher, T. Büchner, P. Guttmann, S. Werner, G. Schneider and J. Kneipp, Nanoscale Adv., 2019, 1, 29372945.

12 J. Kneipp, H. Kneipp, M. McLaughlin, D. Brown and K. Kneipp, Nano Lett., 2006, 6, 2225-2231.

13 G. P. Szekeres and J. Kneipp, Analyst, 2018, 143, 6061-6068.

14 B. Fazio, C. D’Andrea, A. Foti, E. Messina, A. Irrera, M. G. Donato, V. Villari, N. Micali, O. M. Marago and P. G. Gucciardi, Sci. Rep., 2016, 6, 26952.

15 M. Aioub and M. A. El-Sayed, J. Am. Chem. Soc., 2016, 138, 1258-1264.

16 A. Huefner, W. L. Kuan, K. H. Muller, J. N. Skepper, R. A. Barker and S. Mahajan, ACS Nano, 2016, 10, 307-316.

17 D. Drescher, P. Guttmann, T. Büchner, S. Werner, G. Laube, A. Hornemann, B. Tarek, G. Schneider and J. Kneipp, Nanoscale, 2013, 5, 9193-9198.

18 V. Zivanovic, S. Seifert, D. Drescher, P. Schrade, S. Werner, P. Guttmann, G. P. Szekeres, S. Bachmann, G. Schneider, C. Arenz and J. Kneipp, ACS Nano, 2019, 13, 93639375.

19 P. C. Lee and D. Meisel, J. Phys. Chem., 1982, 86, 33913395.

20 G. P. Szekeres and J. Kneipp, Front. Chem., 2019, 7, 30.

21 S. Tenzer, D. Docter, J. Kuharev, A. Musyanovych, V. Fetz, R. Hecht, F. Schlenk, D. Fischer, K. Kiouptsi, C. Reinhardt, K. Landfester, H. Schild, M. Maskos, S. K. Knauer and R. H. Stauber, Nat. Nanotechnol., 2013, 8, 772-781.

22 D. N. Perkins, D. J. C. Pappin, D. M. Creasy and J. S. Cottrell, Electrophoresis, 1999, 20, 3551-3567.

23 D. W. Huang, B. T. Sherman and R. A. Lempicki, Nucleic Acids Res., 2008, 37, 1-13.

24 D. W. Huang, B. T. Sherman and R. A. Lempicki, Nat. Protoc., 2009, 4, 44-57.

25 D. Szklarczyk, A. Franceschini, S. Wyder, K. Forslund, D. Heller, J. Huerta-Cepas, M. Simonovic, A. Roth,
A. Santos, K. P. Tsafou, M. Kuhn, P. Bork, L. J. Jensen and C. von Mering, Nucleic Acids Res., 2015, 43, D447-D452.

26 G. Schneider, P. Guttmann, S. Heim, S. Rehbein, F. Mueller, K. Nagashima, J. B. Heymann, W. G. Muller and J. G. McNally, Nat. Methods, 2010, 7, 985-987.

27 P. H. C. Eilers, Anal. Chem., 2003, 75, 3631-3636.

28 G. P. Szekeres, M. Montes-Bayón, J. Bettmer and J. Kneipp, Anal. Chem., 2020, 92, 8553-8560.

29 L. Vroman, A. L. Adams, G. C. fischer and P. C. Munoz, Blood, 1980, 55, 156-159.

30 V. P. Zhdanov and N. J. Cho, Math. Biosci., 2016, 282, 8290.

31 S. H. Brewer, W. R. Glomm, M. C. Johnson, M. K. Knag and S. Franzen, Langmuir, 2005, 21, 9303-9307.

32 U. Rescher and V. Gerke, J. Cell Sci., 2004, 117, 2631-2639.

33 M. Klobucar, M. Sedic, P. Gehrig, J. Grossmann, M. Bilic, L. Kovac-Bilic, K. Pavelic and S. Kraljevic Pavelic, Biochim. Biophys. Acta, 2016, 1862, 1938-1954.

34 B. J. Galletta, O. L. Mooren and J. A. Cooper, Curr. Opin. Biotechnol., 2010, 21, 604-610.

35 S. M. Smith, M. Baker, M. Halebian and C. J. Smith, Front. Mol. Biosci., 2017, 4, 72.

36 S. L. Schmid, Annu. Rev. Biochem., 1997, 66, 511-548.

37 J. A. Johnston, C. L. Ward and R. R. Kopito, J. Cell Biol., 1998, 143, 1883-1898.

38 M. J. Bollong, M. Pietilä, A. D. Pearson, T. R. Sarkar, I. Ahmad, R. Soundararajan, C. A. Lyssiotis, S. A. Mani, P. G. Schultz and L. L. Lairson, Proc. Natl. Acad. Sci. U. S. A., 2017, 114, E9903.

39 J. Xu, R. Camfield and S. M. Gorski, J. Cell Sci., 2018, 131, jcs215210.

40 F. Ding, Y. Li, J. Liu, L. Liu, W. Yu, Z. Wang, H. Ni, B. Liu and P. Chen, Int. J. Nanomed., 2014, 9, 4317-4330.

41 C. Thery, M. Boussac, P. Veron, P. Ricciardi-Castagnoli, G. Raposo, J. Garin and S. Amigorena, J. Immunol., 2001, 166, 7309-7318.

42 K. Kai, O. Nagano, E. Sugihara, Y. Arima, O. Sampetrean, T. Ishimoto, M. Nakanishi, N. T. Ueno, H. Iwase and H. Saya, Cancer Sci., 2009, 100, 2275-2282.

43 S. N. Saldanha, R. Kala and T. O. Tollefsbol, Exp. Cell Res., 2014, 324, 40-53.

44 M. R. Bhonde, M. L. Hanski, J. Budczies, M. Cao, B. Gillissen, D. Moorthy, F. Simonetta, H. Scherubl, M. Truss, C. Hagemeier, H. W. Mewes, P. T. Daniel, M. Zeitz and C. Hanski, J. Biol. Chem., 2006, 281, 86758685.

45 G. Maiorano, S. Sabella, B. Sorce, V. Brunetti, M. A. Malvindi, R. Cingolani and P. P. Pompa, ACS Nano, 2010, 4, 7481-7491.

46 Z. Yang and H.-R. Xiong, in Biomedical Tissue Culture, ed. L. Ceccherini-Nelli and B. Matteoli, IntechOpen, online, 1st edn, 2012, ch. 1, pp. 3-18.

47 M. K. Sakamoto, S. Mima, T. Kihara and T. Tanimura, Anat. Rec., Part A, 2004, 279, 652-663.

48 E. Falcieri, P. Gobbi, A. Cataldi, L. Zamai, I. Faenza and M. Vitale, Histochem. J., 1994, 26, 754-763. 
49 S. B. Berman, F. J. Pineda and J. M. Hardwick, Cell Death Differ., 2008, 15, 1147-1152.

50 R. J. Youle and M. Karbowski, Nat. Rev. Mol. Cell Biol., 2005, 6, 657-663.

51 C. L. Yen, M. H. Mar, C. N. Craciunescu, L. J. Edwards and S. H. Zeisel, J. Nutr., 2002, 132, 1840-1847.

52 A. S. Rambold, B. Kostelecky, N. Elia and J. LippincottSchwartz, Proc. Natl. Acad. Sci. U. S. A., 2011, 108, 1019010195.

53 W. J. Lee, M. H. Chien, J. M. Chow, J. L. Chang, Y. C. Wen, Y. W. Lin, C. W. Cheng, G. M. Lai, M. Hsiao and L. M. Lee, Sci. Rep., 2015, 5, 10420.

54 C. Wu, C. Wang, J. Zheng, C. Luo, Y. Li, S. Guo and J. Zhang, ACS Nano, 2015, 9, 7913-7924.

55 S. Elmore, Toxicol. Pathol., 2007, 35, 495-516.

56 S. Kapishnikov, A. Weiner, E. Shimoni, P. Guttmann, G. Schneider, N. Dahan-Pasternak, R. Dzikowski, L. Leiserowitz and M. Elbaum, Proc. Natl. Acad. Sci. U. S. A., 2012, 109, 11188-11193.

57 L. Haversen, J. P. Sundelin, A. Mardinoglu, M. Rutberg, M. Stahlman, U. Wilhelmsson, L. M. Hulten, M. Pekny, P. Fogelstrand, J. F. Bentzon, M. Levin and J. Boren, Sci. Rep., 2018, 8, 16973.

58 Y. Cheng, W. Boll, T. Kirchhausen, S. C. Harrison and T. Walz, J. Mol. Biol., 2007, 365, 892-899.
59 H. T. McMahon and E. Boucrot, Nat. Rev. Mol. Cell Biol., 2011, 12, 517-533.

60 C. J. Merrifield and M. Kaksonen, Cold Spring Harbor Perspect. Biol., 2014, 6, a016733.

61 S. Palvai, M. M. Kuman, P. Sengupta and S. Basu, ACS Omega, 2017, 2, 7868-7880.

62 D. A. Kuhn, D. Vanhecke, B. Michen, F. Blank, P. Gehr, A. Petri-Fink and B. Rothen-Rutishauser, Beilstein J. Nanotechnol., 2014, 5, 1625-1636.

63 J. R. Marques, R. R. da Fonseca, B. Drury and A. Melo, Int. J. Mol. Sci., 2010, 11, 4673-4686.

64 R. Srivastava, ChemistrySelect, 2017, 2, 2789-2796.

65 S. Dominguez-Medina, J. Blankenburg, J. Olson, C. F. Landes and S. Link, ACS Sustainable Chem. Eng., 2013, 1, 833-842.

66 G. Orts-Gil, K. Natte, D. Drescher, H. Bresch, A. Mantion, J. Kneipp and W. Österle, J. Nanopart. Res., 2011, 13, 15931604.

67 S. Salgin, U. Salgin and S. Bahadir, Int. J. Electrochem. Sci., 2012, 7, 12404-12414.

68 Z. Movasaghi, S. Rehman and I. U. Rehman, Appl. Spectrosc. Rev., 2007, 42, 493-541.

69 L. M. Almehmadi, S. M. Curley, N. A. Tokranova, S. A. Tenenbaum and I. K. Lednev, Sci. Rep., 2019, 9, 12356. 